\title{
Anatomía de seis especies de helechos del género Dryopteris (Dryopteridaceae) de México
}

\author{
Victoria Hernández ${ }^{1}$, Teresa Terrazas ${ }^{1}$ \& Guillermo Angeles ${ }^{2}$ \\ Colegio de Postgraduados, Programa de Botánica, Montecillo, 56230. Estado de México, México. winchi@colpos.mx \\ Instituto de Ecología, A.C., Departamento Productos Forestales Km. 2.5 antigua carretera a Coatepec No. 351, \\ Congregación El Haya, Xalapa 91070, Veracruz, México
}

Recibido 13-I-2003. Corregido 27-XI-2005. Aceptado 02-V-2006.

\begin{abstract}
Anatomy of six species of Dryopteris ferns (Dryopteridaceae) from Mexico. Rhizome and foliar anatomy of the Mexican Dryopteris Adans. species were studied and compared with other Dryopteridaceae and other fern families to identify anatomical features with diagnostic value. The anatomy of rhizome, stipe, and blade is similar in species of the Dryopteris patula complex. The cells with un-lignified, thickened wall, with cap or U-shape around the meristeles belong to the collenchyma, in contrast with other fern families. Dryopteris wallichiana (Spreng.) Hyl. is anatomically distinguished from the other studied species by having more layers of sclerenchyma and meristeles on the stipe, and by the lack of sclereid nests on the rhizome. Dryopteris rossii C. Chr. and D. maxonii Underw. \& C. Chr. are characterized by the presence of crystals on the periphery of rhizome nests. D. maxonni and D. wallichiana lack blade glands. Rev. Biol. Trop. 54 (4): 1157-1169. Epub 2006 Dec. 15.
\end{abstract}

Key words: anatomy, blade, Dryopteris, Dryopteridaceae, Mexico, rhizome, stipe.

Los estudios anatómicos de pteridofitas han permitido comparar y separar familias, géneros y especies (Ogura 1972, Lin y DeVol 1977, 1978, Moran 1987). White (1974) menciona la importancia de investigar la anatomía de los helechos enfocada a los meristemos del vástago, tejido vascular y tipos de estela, así como la ontogenia de los estomas. Para el rizoma la presencia o ausencia de nidos de esclereidas, las meristelas rodeadas o no por una banda esclerosada y la presencia de células de mucílago son caracteres importantes para distinguir entre especies (Sen y Mittra 1966, Zlotnik 1991, Pacheco y Moran 1999). Otros autores han encontrado caracteres anatómicos diagnósticos en el pecíolo como son el número, forma, arreglo y configuración del xilema de las meristelas y la presencia de la banda esclerosada alrededor de éstos. En la lámina se considera, para distinguir entre especies la forma y tamaño de las células epidérmicas; los tipos de estomas, su posición, tamaño y abundancia; la composición del mesofilo y la presencia de colénquima asociado a las meristelas (Riba 1967, Velasco 1971, Lin y DeVol 1977, 1978, Velásquez 1983, Moran 1987, Saiki et al. 1988, Guantay y Hernández 1990, Kramer y Green 1990, Zlotnik 1991, Herrera 1993, Aguilar et al. 1994, Pérez-García et al. 1995, Zavaro et al. 1995, Mendoza et al. 1998, Zhang y Nooteboom 1998, Silva et al. 2001). Recientemente, la microscopía electrónica de barrido permitió reconocer la presencia de elementos de vaso en algunas familias, géneros y especies de helechos (Carlquist y Schneider 1997, 1999, 2000, 2001, Li et al. 1999, Schneider y Carlquist 1998a, b, c, 1999a, b, 2000).

En la familia Dryopteridaceae se ha estudiado la anatomía del tallo (rizoma) y es descrita como dictiostela al igual que en Davalliaceae y Lomariopsidaceae (Kramer y Green 1990). Además, Li et al. (2000) citan elementos de 
vasos en las raíces y el rizoma de Dryopteris crassirhizoma Nakai, 1920.

En México, el género Dryopteris Adans., 1763 está representado por 12 especies (Riba y Pérez-García 1999). En el género se reconocen varios complejos, uno de ellos es el complejo Dryopteris patula (Sw.) Underw., 1893. Recientemente Mickel y Smith (2004) señalan que D. rosea (Fourn.) Mickel \& Beitel, 1988, es sinónimo de D. cinnamomea (Cav.) C. Chr., 1911; sin embargo, Hernández (2003) muestra con base en un análisis de caracteres morfológicos, que las especies del complejo se pueden separar como válidas. Las especies de Dryopteris habitan bosques de encino, pino-encino, pino y otras coníferas, mesofilo de montaña y tropical caducifolio, así como matorral subtropical. Crecen en suelos rocosos de origen volcánico, oscuro arcilloso con abundante materia orgánica y poco profundos, en altitudes que van desde los 1010 hasta $3100 \mathrm{msnm}$. Generalmente están asociadas con Elaphoglossum Schott ex J. Sm., 1841, Selaginella P. Beauv., 1804 y líquenes (Rzedowski 1978, Tryon y Tryon 1982). Los estudios monográficos del género Dryopteris y florísticos en México detallan los caracteres morfológicos vegetativos y reproductivos (Knobloch y Correll 1962, Smith 1981, Mickel y Beitel 1988, Díaz-Barriga y PalaciosRios 1992, Mickel 1992, Palacios-Rios 1992, Moran 1995, Riba y Pérez-García 1999), pero no presentan atributos anatómicos que permitan comparar las especies presentes en México. El objetivo del presente estudio fue comparar la anatomía del rizoma, pecíolo y lámina de seis especies de Dryopteris en México, en busca de caracteres diagnósticos con valor taxonómico para la delimitación de las especies del complejo Dryopteris patula y además cotejar los caracteres anatómicos de Dryopteris con los de otros géneros de Dryopteridaceae y otras familias de pteridofitas.

\section{MATERIALES Y MÉTODOS}

Parte del material estudiado se recolectó en los estados de Guerrero, Estado de México,
Michoacán, Morelos y Veracruz (V. Hernández et al. ejemplares depositados en CHAPA), el resto procede de ejemplares de diferentes herbarios (Cuadro 1). El material vivo se separó en rizoma, pecíolo y lámina y se fijaron en FAA (formaldehído, ácido acético glacial y alcohol etílico). El material de herbario (pecíolo, lámina) se hidrató en una solución de $\mathrm{NaOH}$ al $5 \%$ por 5 min y después se colocó en FAA durante $24 \mathrm{~h}$.

Se realizaron cortes transversales a mano del pecíolo y la mitad de los cortes se aclararon con hipoclorito de sodio (cloro comercial al $50 \%$ ) para eliminar los contenidos. Todos los cortes se deshidrataron en concentraciones de alcohol etílico (50, 70, $95 \%$ ), se tiñeron con safranina-verde fijo y montaron en resina sintética. Además, se realizaron cortes de la lámina y el pecíolo de material herborizado; este material se deshidrató en un cambiador automático Leica TP1020 y se incluyó en parafina. Una vez incluidos en parafina se hicieron cortes transversales y paradermales en un micrótomo rotatorio a $12 \mu \mathrm{m}$ de grosor, se tiñeron con safranina-verde fijo y montaron en resina sintética (Johansen 1940). Los cortes transversales de rizoma (material recolectado) se efectuaron en el micrótomo de deslizamiento a un grosor de $80 \mu \mathrm{m}$; una parte de los cortes se aclararon con hipoclorito de sodio (cloro comercial al $50 \%$ ) durante $24 \mathrm{~h}$, se tiñeron y montaron con la misma técnica usada para los cortes de pecíolo. En cuanto a la lámina del material recolectado, ésta se cortó en el micrótomo rotatorio a un grosor de $20 \mu \mathrm{m}$. Una parte de los cortes se montaron sin teñir en gelatina glicerinada y la otra parte se deshidrató, tiñó y montó con la técnica ya mencionada.

La pinna basal se diafanizó con $\mathrm{NaOH}$ al $5 \%$ durante $24-48 \mathrm{~h}$ y con hipoclorito de sodio (cloro comercial al $50 \%$ ) por $15 \mathrm{~min}$, hasta eliminar por completo los pigmentos. Se deshidrató y tiñó con safranina en alcohol al $95 \%$ durante $15 \mathrm{~min}$, se continuó la deshidratación con alcohol absoluto por $10 \mathrm{~min}$, xileno-aceite de clavo (2:1) por $1 \mathrm{~h}$ y xileno puro por $2 \mathrm{~h}$ para montar en resina sintética (Gardner 1975, D’Ambrogio 1986).

Para la elaboración de las descripciones anatómicas del rizoma, pecíolo y lámina, se 
CUADRO 1

Material estudiado de seis especies de Dryopteris. Colector(es), estado de origen y herbario

TABLE 1

Material studied of six species of Dryopteris. Collector(s), state of origin, and herbarium

Especies

D. cinnamomea (Cav.) C. Chr.
Colector (es)

R. McVaugh y W.N. Koelz 214

R. Spellenberg y D. Jewell s.n.

A.Mondragón 200

F. García 39

Y. Herrera y J. Rzedowski 142

V. Hernández y E. Martínez 371

J.D. Galván 2543

D.E. Breedlove 39287

V. Hernández y S. Zamudio 446

Almazán et al. 820

E. Callejas et al. 41

G. Flores, R. Ramírez y O. Tellez 2203

C.R. Orcutt 3995

A. Sharp 441655

J. Rzedowski 45740

E. Argüelles 1714

T.R. van Devender et al. 98-1038

V. Hernández et al. 347

D. maxonii Underw. \& C. Chr.

D. patula (Sw.) Underw.

V. Hernández y E. Martínez 357

E. Ventura A. y E. López 2560

V. Hernández y E. Martínez 368

J.R. Pérez 2a

J.T. Mickel 1260

V. Hernández y P.F. Franco 460

V. Hernández et al. 340, 348

D. rosea (E. Fourn.) Mickel \& Beitel V. Hernández et al. 430, 441, 440, 428, 429

J. Rzedowski 18525

F. Lorea 2496

H. Díaz-Barriga 6425

J.T. Mickel y S.W. Leonard 4428A

V. Hernández et al. 338

D. rossii C. Chr.
V. Hernández y E. Martínez 354

V. Hernández y E. Martínez 395

F. Lorea 4681
Estado

Aguascalientes

Chihuahua

Distrito Federal

Distrito Federal

Durango

Estado de México

Guanajuato

Jalisco

Michoacán

Michoacán

Morelos

Nayarit

Puebla

Puebla

Querétaro

Querétaro

Sonora

Veracruz

Estado de México

Chiapas

Estado de México

Guanajuato

Oaxaca

Veracruz

Veracruz

Guerrero

Guerrero

Guerrero

Michoacán

Oaxaca

Veracruz

Estado de México

Estado de México

Guerrero
Herbario

IEB

MEXU

MEXU

ENCB

ENCB

CHAPA

ENCB

MEXU

CHAPA

IEB

MEXU

MEXU

MEXU

MEXU

MEXU

MEXU

MEXU

CHAPA

CHAPA

ENCB

CHAPA

IEB

ENCB

CHAPA

CHAPA

CHAPA

ENCB

IEB

IEB

MEXU

CHAPA

CHAPA

CHAPA

IEB 
CUADRO 1 (Continuación)

Material estudiado de seis especies de Dryopteris. Colector(es), estado de origen y herbario

TABLE 1 (Continued)

Material studied of 6 species of Dryopteris. Collector(s), state of origin, and herbarium

\begin{tabular}{|c|c|c|c|}
\hline \multirow[t]{7}{*}{ Especies } & Colector (es) & Estado & Herbario \\
\hline & V. Hernández et al. 438 & Guerrero & CHAPA \\
\hline & V. Hernández et al. 439 & Guerrero & CHAPA \\
\hline & J.A. García 5456 & Jalisco & ENCB \\
\hline & V. Hernández y S. Zamudio 459 & Michoacán & CHAPA \\
\hline & V. Hernández y S. Zamudio 444, 448 & Michoacán & CHAPA \\
\hline & C. Feddema 2661 & Nayarit & ENCB \\
\hline \multirow[t]{3}{*}{ D. wallichiana (Spreng.) Hyl. } & V. Hernández et al. 419 & Guerrero & CHAPA \\
\hline & V. Hernández et al. 334a & Veracruz & CHAPA \\
\hline & V. Hernández et al. 336 & Veracruz & CHAPA \\
\hline
\end{tabular}

generaron y cuantificaron caracteres del tejido dérmico, fundamental y vascular. En el pecíolo se cuantificó el largo y ancho de las células epidérmicas, el ancho y largo de las células del esclerénquima y el número de estratos; además se contó el número de meristelas y el número de hileras de células del periciclo. En la lámina se midieron las glándulas, el largo de las células oclusivas y se contó el número de hileras de parénquima en empalizada y esponjoso. Se hicieron 20 mediciones o conteos por carácter señalado por individuo, con la ayuda del programa Image Pro-Plus ${ }^{\circledR}$ versión 3.1 adaptado a una cámara de video Hitachi KP-D51 y a un microscopio Olympus BX50. Únicamente se sintetizan los valores cuantitativos a través de la media o la moda, debido a que el número de muestra fue escaso y no permitió realizar ningún análisis estadístico. La terminología utilizada en las descripciones se basó en la literatura revisada para pteridofitas (Sen y Mittra 1966, Ogura 1972, Lin y DeVol 1977, 1978, Sen y Hennipman 1981, Zlotnik 1991, Herrera 1993).

\section{RESULTADOS}

Descripción anatómica del rizoma: $\mathrm{La}$ epidermis es simple con células de diferente forma, desde cuadrada, rectangular a poligonal. Las especies D. cinnamomea, D. patula, D. rosea y D. rossii C. Chr., 1922 presentan glándulas y escamas en el margen; sin embargo, D. maxonii Underw. \& C. Chr., 1911 y D. wallichiana (Spreng.) Hyl., 1953 carecen de glándulas. La corteza primaria está constituida por parénquima y grupos de esclereidas denominados nidos (Fig. 1). Las células del parénquima son isodiamétricas con paredes delgadas y pequeños espacios intercelulares y comúnmente con contenidos de color oscuro. Los nidos de esclereidas sin espacios intercelulares entre ellas caracterizan a $D$. cinnamomea y D. rosea (Fig. 2), mientras que en D. patula se observan espacios intercelulares abundantes. Se detectaron cristales en la periferia de los nidos de esclereidas en $D$. rossii y $D$. maxonii (Fig. 3) y en $D$. wallichiana no se observaron los nidos de esclereidas. La estela es del tipo dictiostela; el número de meristelas es seis en D. rosea y nueve en D. maxonii (Cuadro 2). Alrededor de las meristelas se presenta la endodermis, distintiva por las células rectangulares y la banda de Caspari en sus paredes radiales. La estela está formada por el periciclo con un máximo de tres hileras de células (Cuadro 2), el xilema está constituido por elementos vaso 


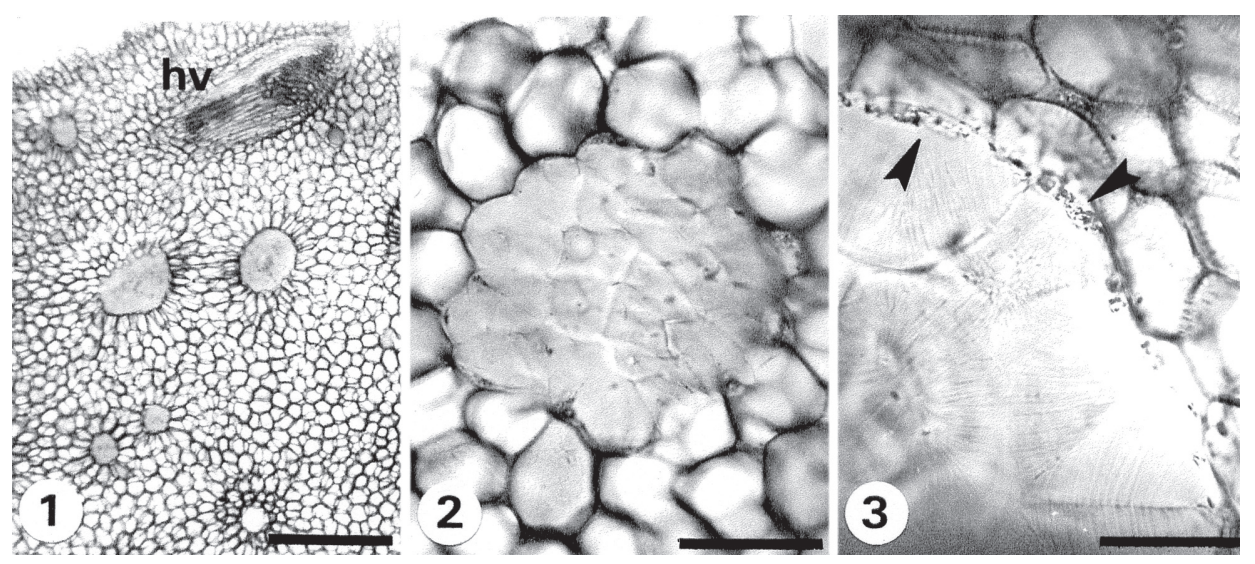

Fig. 1-3. Cortes transversales de rizoma. 1. Dryopteris rosea (V. Hernández et al. 338) tejido fundamental con meristelas y nidos. 2. D. maxonii (V. Hernández et al. 357) nido de esclereidas. 3. D. rossii (V. Hernández et al. 354) cristales en la periferia de los nidos de esclereidas. Escala: $1=500 \mu \mathrm{m}, 2=100 \mu \mathrm{m}, 3=50 \mu \mathrm{m}, \mathrm{cr}=$ cristales, $\mathrm{m}=$ meristelas, $\mathrm{n}=$ nidos de esclereidas.

Fig. 1-3. Transverse sections of the rhizome. 1. Dryopteris rosea (V. Hernández et al. 338) fundamental tissue with meristeles and nests. 2. D. maxonii (V. Hernández et al. 357) sclereid nests. 3. D. rossii (V. Hernández et al. 354) crystals on the periphery of the sclereids nests. Scale: $1=500 \mu \mathrm{m}, 2=100 \mu \mathrm{m}, 3=50 \mu \mathrm{m}$, cr= crystals, $\mathrm{m}=$ meristeles, $\mathrm{n}=$ sclereid nests.

CUADRO 2

Caracteres anatómicos del rizoma en las especies estudiadas de Dryopteris

TABLE 2

Rhizome anatomical features of the species of Dryopteris studied

\section{Caracteres/Especie}

Número de hileras del periciclo

Número de meristelas

Nidos de esclereidas

Cristales alrededor de los nidos de esclereidas
D. cinnamomea

(2) -3

(6) -7

Sí

No

D. maxoni

D. patula

D. rosea

D. rossii

D. wallichiana

Se representa la moda entre paréntesis. / The mode is represented in parenthesis.

(2)-3 1-(2) 2-(3)

(6) -7

6

7-(8)

(8)-9

Sí

Sí

No

Sí

No y el floema por células pequeñas y parénquima floemático con contenidos oscuros, taninos.

\section{Descripción anatómica de pecíolo: El} pecíolo es cilíndrico con un surco en la cara adaxial que se prolonga hacia el raquis (Fig. 4), con escamas en la base y con glándulas, excepto en $D$. maxonii y $D$. wallichiana. En el corte transversal, la cutícula es delgada y lisa en todas las especies estudiadas. La epidermis es simple (Fig. 5), con células de pared delgada, cuadradas a rectangulares que varían en $D$. patula de 6-17 $\mu \mathrm{m}$ de longitud por 6-20 $\mu \mathrm{m}$ de ancho hasta 11-34 $\mu \mathrm{m}$ de longitud por 11-29 $\mu \mathrm{m}$ de ancho en D. rossii (Cuadro 3).

La corteza está compuesta por esclerénquima y parénquima (Fig. 5). En la parte externa subyacente a la epidermis se observa el esclerénquima, constituido de varias hileras de células que fluctúan de cuatro en $D$. cinnamomea y $D$. rosea hasta nueve en $D$. wallichiana (Cuadro 3); de forma cuadrada, pentagonal a poligonal, con paredes gruesas y sin espacios intercelulares. Las células del parénquima son isodiamétricas con paredes delgadas y se observan espacios intercelulares pequeños. La parte 
CUADRO 3

Caracteres anatómicos del peciolo de las especies de Dryopteris

TABLE 3

Anatomical features of the stipe in Dryopteris species

\begin{tabular}{|c|c|c|c|c|c|c|}
\hline Caracteres/Especie & D. cinnamomea & D. maxonii & D. patula & D. rosea & D. rossii & D. wallichiana \\
\hline $\begin{array}{l}\text { Largo y ancho de las células } \\
\text { epidérmicas }(\mu \mathrm{m})\end{array}$ & $9-18 \times 11-21$ & $6-18 \times 7-22$ & $6-17 \times 6-20$ & $9-25$ X 12-41 & $11-34$ X 11-29 & $9-14 \times 9-19$ \\
\hline $\begin{array}{l}\text { Número de hileras del } \\
\text { esclerénquima }\end{array}$ & $2-(4)-6$ & $(6)-10$ & $3-(6)-7$ & (4)-7 & $4-(5)-8$ & (9)-11 \\
\hline $\begin{array}{l}\text { Largo y ancho de las células } \\
\text { del esclerénquima }(\mu \mathrm{m})\end{array}$ & $8-19 \times 12-30$ & $8-19 \times 9-26$ & $8-18 \times 8-24$ & $12-40 \times 23-50$ & $13-28 \times 12-42$ & $7-13 \times 8-19$ \\
\hline Número de meristelas & $\begin{array}{c}\mathrm{b}=3-4 \\
\mathrm{~m}=3, \mathrm{r}=2\end{array}$ & $\begin{array}{c}\mathrm{b}=7 \\
\mathrm{~m}=5, \mathrm{r}=5\end{array}$ & $\begin{array}{c}\mathrm{b}=5-7 \\
\mathrm{~m}=5, \mathrm{r}=3\end{array}$ & $\begin{array}{c}\mathrm{b}=5-6 \\
\mathrm{~m}=4, \mathrm{r}=3\end{array}$ & $\begin{array}{c}\mathrm{b}=5-7 \\
\mathrm{~m}=4, \mathrm{r}=2-3\end{array}$ & $\begin{array}{c}\mathrm{b}=8-9 \\
\mathrm{~m}=7-8, \mathrm{r}=7\end{array}$ \\
\hline $\begin{array}{l}\text { Número de hileras del } \\
\text { periciclo }\end{array}$ & $1-3$ & $1-2$ & $1-3$ & $1-3$ & $1-2$ & $1-3$ \\
\hline
\end{tabular}

más interna de la corteza se diferencia en una banda, donde los engrosamientos en las células de ésta, pueden ser en la pared periclinal en forma de casquete y en otras células el engrosamiento se extiende hacia las paredes anticlinales en forma de "U" (Fig. 6). Esta banda uniseriada es distintiva por los depósitos color marrón en la pared engrosada no lignificada (Fig. 7). La endodermis se caracteriza por la forma de las células rectangulares con la banda de Caspari en sus paredes radiales (Fig. 6). El periciclo se constituye por uno a tres estratos de células en cinco especies y sólo $D$. rossii presenta de una a dos hileras de células (Cuadro 3) y con paredes delgadas. El número de meristelas varía a lo largo del pecíolo; por ejemplo en $D$. cinnamomea se presentan de tres a cuatro en la base y dos hacia el raquis (Cuadro 3). El arreglo de las meristelas adquiere forma de arco, con los haces de mayor tamaño hacia la cara adaxial y entre ellos los pequeños. En las meristelas de mayor tamaño el xilema termina en un gancho curvado hacia adentro, denominado hipocampo. Los elementos de vasos con punteaduras escalariformes; el floema se constituye de células pequeñas, con grosor de pared primaria diferente y algunas con contenidos oscuros en el lumen celular. La anatomía del raquis es muy parecida a lo anteriormente descrito; sin embargo, el número de las meristelas se reduce en todas las especies estudiadas. Algunas veces éstos se fusionan como es el caso de $D$. rossii que puede presentar dos o tres.

Patrón de venación y anatomía foliar: El patrón de venación es libre, la vena principal o costa central y las venas secundarias se distinguen por sus diferencias en espesor; las secundarias se bifurcan dos a tres veces terminando muy cerca del margen. La posición de los soros es submarginal y se encuentran en la parte media de las venas. La lámina en vista superficial (corte paradermal) tiene las paredes de las células epidérmicas onduladas (Fig. 8). Los estomas son del tipo polocítico, porque las células oclusivas tienen una célula subsidiaria en forma de herradura en todas las especies estudiadas (Fig. 9). Sin embargo, hay una amplia variación en el tamaño de las células oclusivas, en $D$. wallichia$n a$ de 34-52 $\mu \mathrm{m}$ y $D$. cinnamomea de 53-73 $\mu \mathrm{m}$ (Cuadro 4). En ambas caras se observan glándulas en las especies D. cinnamomea, D. patula, $D$. rosea y $D$. rossii, pero éstas no se detectaron en D. maxonii y D. wallichiana. 

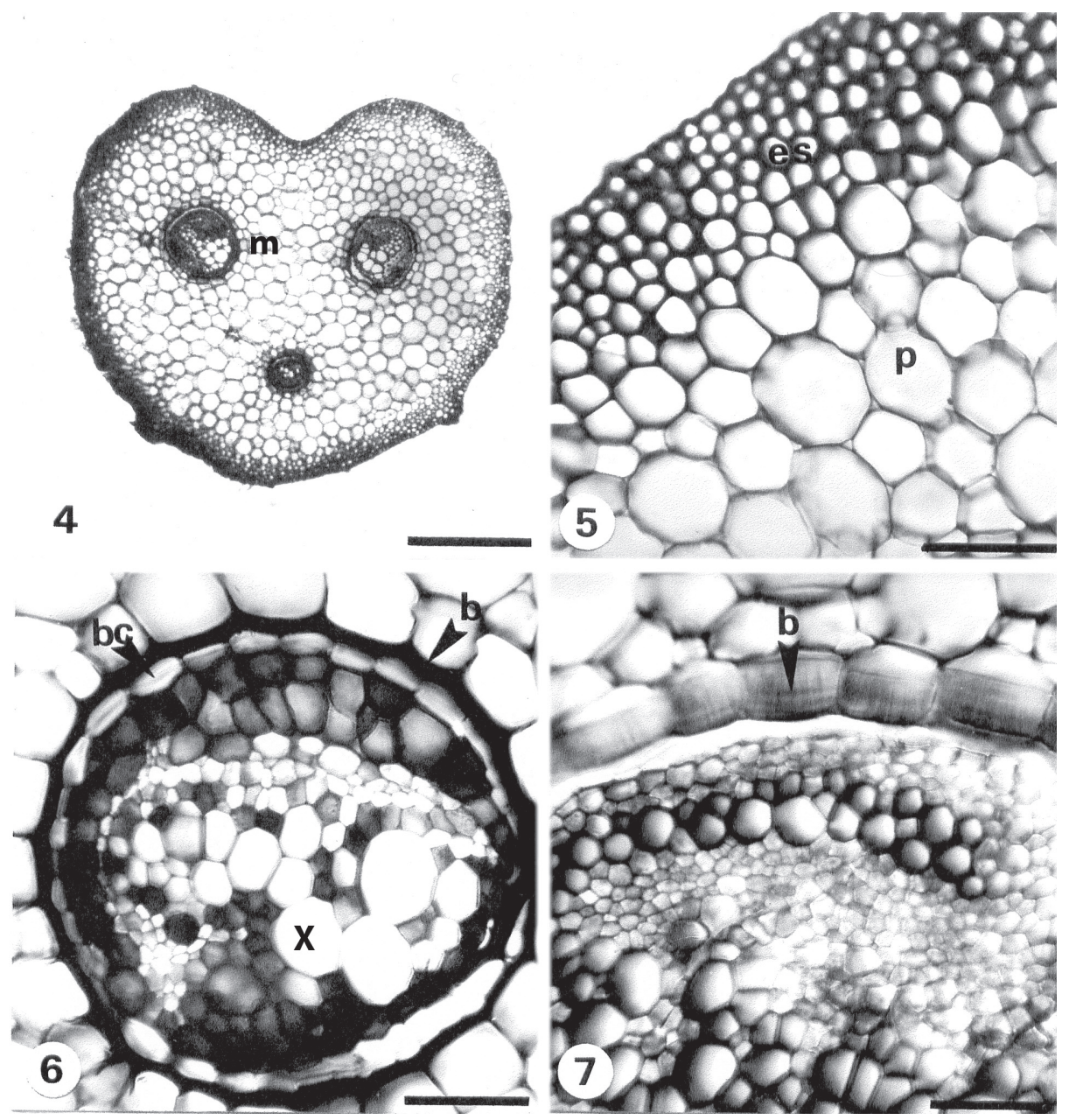

Fig. 4-7. Cortes transversales de pecíolo. 4-5. D. cinnamomea (V. Hernández et al. 343) pecíolo con surco y estele tipo Aspidium. 5. Epidermis, esclerénquima y parénquima. 6. D. cinnamomea (V. Hernández et al. 371) meristela rodeado por una banda de células con contenido marrón y endodermis con banda de Caspari. 7. D. patula (V. Hernández et al. 348) pared interna con engrosamientos en forma de $U$ en las células de la banda que rodea la meristela. Escala: 4, 6, 7= 50 $\mu \mathrm{m}$, $5=100 \mu \mathrm{m}, \mathrm{b}=$ banda, $\mathrm{bc}=$ banda de Caspari, es= esclerénquima, $\mathrm{m}=$ meristela, $\mathrm{p}=$ parénquima, $\mathrm{x}=$ xilema.

Fig. 4-7. Transverse sections of the stipe. 4-5. D. cinnamomea (V. Hernández et al. 343) petiole with furrow and Aspidium stelar type. 5. Epidermis, sclerenchyma and parenchyma. 6. D. cinnamomea (V. Hernández et al. 371) meristele surrounded by a band of cells with a brownish content and endodermis with a Casparian strip. 7. D. patula (V. Hernández et al. 348) inner wall with $U$-shaped thickening in the cells of the band which surrounds the meristele. Scale: 4, 6, 7=50 $\mu \mathrm{m}, 5=100$ $\mu \mathrm{m}, \mathrm{b}=\mathrm{band}, \mathrm{bc}=$ Casparian strip, $\mathrm{es}=$ sclerenchyma, $\mathrm{m}=$ meristele, $\mathrm{p}=$ parenchyma, $\mathrm{x}=\mathrm{xylem}$.

En corte transversal la epidermis de ambas caras tiene células de forma cuadrada a rectangular y con paredes delgadas; los estomas hipostomáticos son elevados, con la excepción de D. patula y D. rosea. El mesofilo está constituido por parénquima en empalizada y esponjoso (Fig. 10). El primero presenta células de forma irregular con abundantes cloroplastos y el número de estratos varía de dos en cinco especies a tres en D. wallichiana (Cuadro 4). El parénquima esponjoso se distingue por presentar células de forma irregular con espacios intercelulares grandes (Fig. 10), el número de hileras varía de dos en $D$. rosea a cuatro en 

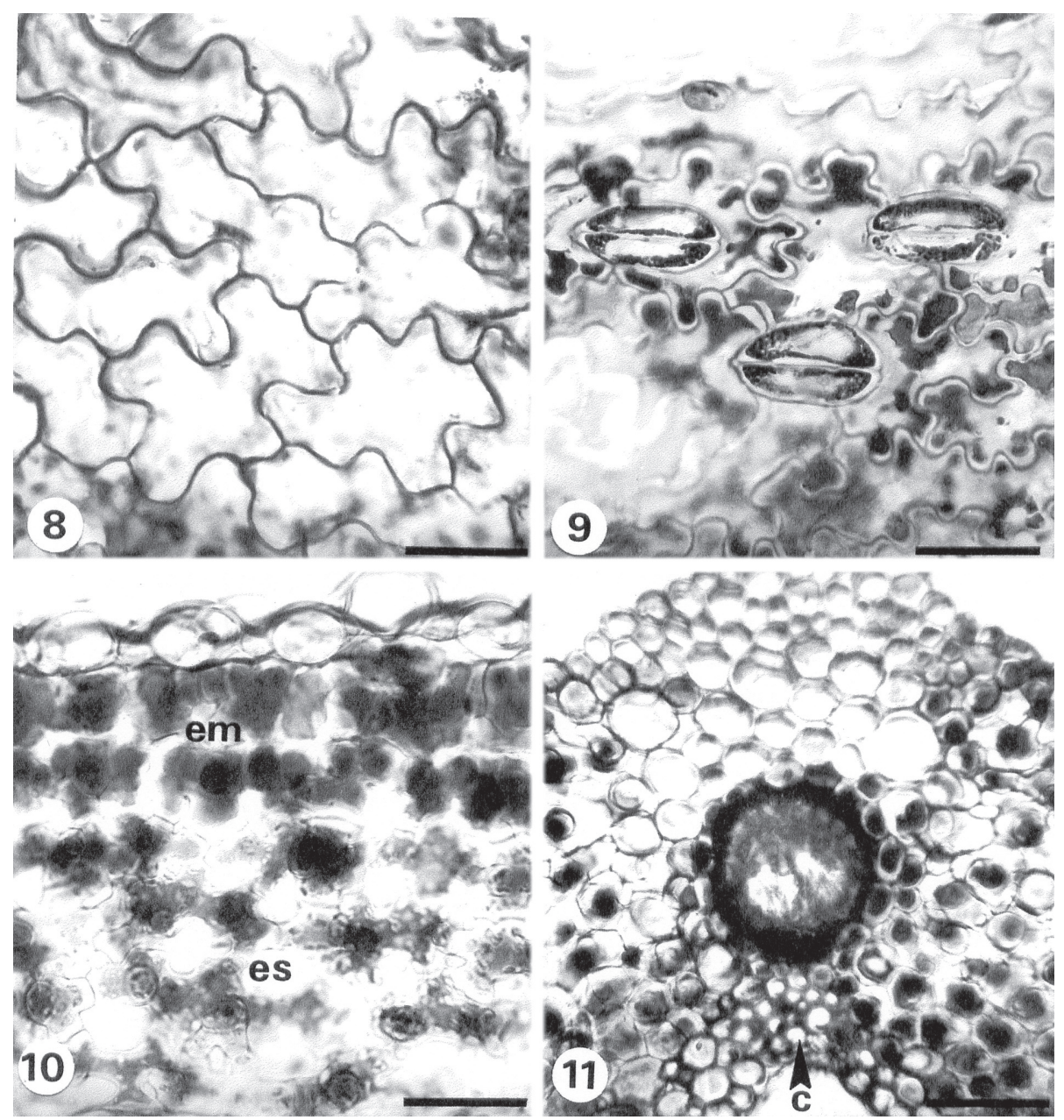

Fig. 8-11. Lámina foliar. 8-9. Cortes paradermales. 8. Dryopteris rosea (J. Rzedowski 18525) células epidérmicas con paredes onduladas. 9. D. patula (J.T. Mickel 1260) estomas polocíticos. 10-11. Cortes transversales. 10. D. patula (V. Hernández et al. 348) parénquima en empalizada de células cortas y esponjoso. 11. D. rossii (V. Hernández et al. 354) células de colénquima cerca de la vena media. Escala: $8-10=50 \mu \mathrm{m}, 1=100 \mu \mathrm{m}, \mathrm{c}=$ colénquima, em= parénquima en empalizada, es= parénquima esponjoso.

Fig. 8-11. Foliar blade. 8-9. Paradermal sections. 8. Dryopteris rosea (J. Rzedowski 18525) epidermal cells with undulated walls. 9. D. patula (J.T. Mickel 1260) polocytic stomata. 10-11. Transverse sections. 10. D. patula (V. Hernández et al. 348) short palisade cells and spongy parenchyma. 11. D. rossii (V. Hernández et al. 354) collenchyma cells near to midrib. Scale: $8-10=50 \mu \mathrm{m}, 11=100 \mu \mathrm{m}, \mathrm{c}=$ collenchyma, em= palisade parenchyma, es $=$ spongy parenchyma.

D. rossii y D. wallichiana (Cuadro 4). El haz vascular de la vena principal y las células asociadas a éste son muy parecidas al arreglo descrito en el pecíolo con una banda de células con paredes engrosadas color marrón; endodermis, periciclo, xilema y floema. Además se observó colénquima formado por dos a tres hileras cerca a la vena principal (Fig. 11).

\section{DISCUSIÓN}

La anatomía del rizoma, pecíolo y lámina resultó ser similar en las seis especies, con algunas excepciones. Respecto a la anatomía del rizoma, D. wallichiana se distingue del resto de las especies estudiadas por la ausencia de nidos de esclereidas en éste; mientras 
CUADRO 4

Caracteres anatómicos de la lámina foliar de las especies de Dryopteris

TABLE 4

Anatomical features of the foliar blade in Dryopteris species

\section{Caracteres/Especie}

Número de capas del parénquima en empalizada

Número de capas del parénquima esponjoso

Tamaño de glándulas $(\mu \mathrm{m})$

Tamaño de células oclusivas $(\mu \mathrm{m})$

Estomas elevados

\begin{abstract}
D. cinnamomea
\end{abstract}

2
$2-(3)$
$60-86$
$53-73$
Sí

D. patula

\author{
2
}

2-(3)

52- 89

43-71

No
D. rosea

(2)-3

2

46-80

38-63

No
D. rossii

2

$$
3-(4)
$$

54-81

$38-67$

Sí
D. maxonii D. wallichiana

$\begin{array}{cc}(2)-3 & (3)-4 \\ 3 & 4 \\ \text { ausentes } & \text { ausentes } \\ 39-51 & 34-52 \\ \text { Sí } & \text { Sí }\end{array}$

Se representa la moda entre paréntesis. / The mode is represented in parenthesis.

D. rossii y D. maxonii se separan por presentar cristales en la periferia de los nidos de esclereidas. Estos caracteres aparentemente podrían ser importantes para delimitar grupos de especies, por lo que se recomienda continuar el estudio anatómico del rizoma de las especies del género Dryopteris y otros géneros de la familia Dryopteridaceae. Los nidos de esclereidas en el rizoma también se han observado en especies del género Campyloneurum C. Presl, 1836 (Zlotnik 1991).

La forma, número y arreglo de los haces vasculares del pecíolo son caracteres importantes para la identificación de familias, géneros y especies de helechos (Lin y DeVol 1977, 1978). Ogura (1972) propuso una clasificación del tipo estelar para pecíolo y raquis; y de acuerdo con ésta, el género Dryopteris presenta el tipo Aspidium. Este tipo consiste de más de tres meristelas arreglados en forma de arco, los dos haces más grandes orientados hacia la cara adaxial y el xilema es de la forma hipocampo con la terminación curvada en gancho; mientras que los otros haces más pequeños son circulares o elípticos como se observó en las seis especies estudiadas. Además del género Dryopteris la estela de tipo Aspidium se encuentra en los géneros Blechnum L., 1753 y Polystichum Roth, 1799. A pesar de la variación en el número meristelas en las especies de Dryopteris, ésta permitió delimitar entre especies; por ejemplo $D$. wallichiana presenta ocho a nueve meristelas en la base y siete hacia el raquis y puede separarse de las otras cinco especies estudiadas con un menor número de meristelas, por ejemplo en $D$. cinnamomea con tres a cuatro en la base y dos hacia el raquis. Además, la familia Dryopteridaceae se caracteriza por presentar tres o más meristelas. Este carácter permite separarla de las familias Aspleniaceae, Athyriaceae y Thelypteridaceae que sólo tienen dos meristelas (Pacheco y Lorea 1985, Palacios-Rios 1992). De acuerdo con Moran (1987), el arreglo de las meristelas es distinto en los géneros de Dryopteridaceae; por ejemplo en Cyrtomium C. Presl, 1930, Dryopteris y Polystichum muestran forma de "C"; este arreglo se confirmó en las especies estudiadas de Dryopteris; mientras en Polybotrya Humb. \& Bonpl. ex Willd., 1810 es muy semejante a la forma de hongo con la base hacia la cara adaxial. En este último género también el número de meristelas es mayor a tres. Una variación similar se ha informado para los géneros Marattia Sw., 1788 (Velasco 1971), Tectaria Cav., 1799 (Velásquez 1983) y Cheilanthes Sw., 1867 (Herrera 1993).

Las seis especies de Dryopteris presentaron en el pecíolo y la lámina una banda alrededor de las meristelas. Las células de esta banda tuvieron paredes engrosadas de color marrón en cortes no aclarados; sin embargo, 
en los cortes donde este contenido se eliminó, se apreció sólo la pared engrosada formando un casquete o en "U". En el género Tectaria la banda se describe como una hilera de la corteza interna con engrosamientos en las paredes radiales (Velásquez 1983), por lo que difiere de lo observado en las especies de Dryopteris donde se encontraron arreglados en forma de "casquete" o en "U". Posiblemente la composición de esta pared sea exclusivamente celulósica debido a que se tiñó de verde con la doble tinción safranina-verde fijo. En la literatura se denomina a esta banda, vaina, anillo o banda esclerosada o esclerótica (Ogura 1972, Lin y DeVol 1977, 1978, Velásquez 1983, Zlotnik 1991). Considerando que en las especies estudiadas de Dryopteris las paredes de las células no estuvieron lignificadas no se debe emplear el término esclerosada para denominar la banda. Ogura (1972) señala que esta banda es una vaina de soporte y la considera como tejido mecánico alrededor de las meristelas del rizoma y pecíolo, por estar constituida por esclereidas con paredes gruesas de color marrón. La banda conservó las paredes no lignificadas en Dryopteris, por ello se interpretó como colénquima, contribuyendo al sostén y proporcionando flexibilidad al pecíolo y lámina (Esau 1976, Niklas 1992); sería interesante confirmar la presencia de colénquima en otras especies de helechos, debido a que también la banda se ha observado en las familias Blechnaceae, Davalliaceae, Polypodiaceae, Oleandraceae (Lin y DeVol 1977, 1978) y Lomariopsidaceae (Rojas, en prep.). Los contenidos color marrón en la células de la banda se observaron en las seis especies estudiadas $\mathrm{y}$, seguramente, corresponden a los taninos descritos para otras especies de pteridofitas (Bell 1951). A pesar de pertenecer a la familia Dryopteridaceae el género Polybotrya no presenta esta banda en el pecíolo y la lámina; sin embargo, Moran (1987) menciona la presencia de un anillo o vaina de esclerénquima formado por varias hileras de fibras en el rizoma y las raíces de Polybotrya. En las especies estudiadas de Dryopteris no se observó dicha banda asociada a las meristelas en el rizoma. Por lo anterior, puede señalarse que la banda es común en la familia Dryopteridaceae; sin embargo, esto no significa que todos los géneros y todos los órganos de la planta la presentan. Se sugiere estudiar la anatomía de los seis taxa restantes de Dryopteris en México y de otras regiones para corroborar su presencia en los diferentes órganos.

Las especies estudiadas de Dryopteris presentaron un patrón de venación libre y simple semejante a Marattia y Cheilanthes (Velasco 1971, Herrera 1993); sin embargo, en los géneros Campyloneurum, Microgramma C. Presl, 1836, Niphidium J. Sm., 1875 y Pleopeltis Humb. \& Bonpl. ex Willd., 1810, la venación es reticulada con areolas (Zlotnik 1991) y en Polybotrya puede ser libre o anastomosada (Moran 1987). El mesofilo estuvo formado por parénquima en empalizada y esponjoso en las especies estudiadas, similar a lo informado para especies de Marattia, Pleopeltis, Cheilanthes, Elaphoglossum y Woodwardia J. E. Sm., 1793 (Velasco 1971, Lucansky 1981, Zlotnik 1991, Guantay y Hernández 1990, Herrera 1993). En los helechos acuáticos los espacios intercelulares suelen ser más grandes y abundantes; a veces las células del parénquima en empalizada tienen forma de "H" y en el esponjoso las células son redondas; en otras especies sólo se menciona como mesofilo (Ogura 1972). La anatomía de la lámina de las especies de Dryopteris estudiadas es diferente a la del género Polybotrya donde el parénquima en empalizada está ausente, sólo presenta células de parénquima cilíndricas y grandes espacios intercelulares; en el lado adaxial tiene brazos de colénquima (Moran 1987); en los géneros Campyloneurum, Microgramma, Niphidium y Tectaria sólo tienen parénquima esponjoso (Velásquez 1983, Zlotnik 1991). Se confirma la presencia de colénquima en la lámina de las especies de Dryopteris, cerca de la vena principal en el cara abaxial. En Asplenium L., 1753, el colénquima se informó también alrededor de las venas, formando una conexión con la hipodermis (Chaerle y Viane 2004).

En conclusión, los caracteres anatómicos de rizoma, pecíolo y lámina de las seis especies 
de Dryopteris resultaron ser homogéneos y proporcionan por sí mismos poca información para separar las especies. Sin embargo, las diferencias anatómicas encontradas en los dos órganos deberán corroborarse al estudiar en otras especies del género Dryopteris tanto a nivel nacional como mundial para confirmar valor diagnóstico.

\section{AGRADECIMIENTOS}

Se agradece a los curadores de CHAPA, ENCB, IEB, MEXU por permitirnos remover material para realizar este estudio. Al CONACyT por la beca otorgada a VHH (No. de registro: 162688) para realizar estudios de Maestría y al Colegio de Postgraduados por las facilidades. A René Hernández-Gómez, Pedro Franco H., Enrique Martínez V., Valentín J. Reyes H. y Cesáreo Catalán-Hevarístico por su valiosa ayuda en la recolecta de campo y a dos revisores anónimos por sus comentarios favorables y sugerencias.

\section{RESUMEN}

Se estudió la anatomía del rizoma y hoja de especies mexicanas de Dryopteris Adans. y se comparó con la información disponible para Dryopteridaceae y otras familias de helechos en busca de caracteres anatómicos con valor diagnóstico. La anatomía de rizoma, pecíolo y lámina es similar en las especies del complejo Dryopteris patula. Las células con pared engrosada, no lignificada y en forma de casquete o de " $U$ " presentes alrededor de las meristelas corresponden a colénquima, a diferencia de lo informado para otras familias de helechos. Dryopteris wallichiana (Spreng.) Hyl. se distingue anatómicamente de las otras especies estudiadas por presentar un mayor número de capas de esclerénquima y de meristelas en el pecíolo, además de carecer de nidos de esclereidas en el rizoma. Dryopteris rossii y D. maxonii se caracterizan por la presencia de cristales en la periferia de los nidos en el rizoma. Las glándulas en la lámina están ausentes en $D$. maxonii y D. wallichiana.

Palabras clave: anatomía, lámina, Dryopteris, Dryopteridaceae, México, rizoma, pecíolo.

\section{REFERENCIAS}

Aguilar-Pérez, C., L. Pacheco \& L. Zelaya-Molina. 1994. Estudio anatómico de Diplazium plantaginifolium (L.) Urban, D. ternatum y D. striatum (L.) Presl., p. 903. In VII Congreso Latinoamericano de Botánica. Mar del Plata, Buenos Aires, Argentina.

Bell, P.R. 1951. Studies in the genus Elaphoglossum. III. Anatomy of the rhizome and frond. Ann. Bot. 15: 347-357.

Carlquist, S. \& E.L. Schneider. 1997. SEM studies on vessels in ferns 1. Woodsia obtusa. Amer. Fern J. 87: $1-8$.

Carlquist, S. \& E.L. Schneider. 1999. SEM studies on vessels in ferns. 12. Marattiaceae, with comments on vessel patterns in Eusporangiate ferns. Amer. J. Bot. 86: 457-464.

Carlquist, S. \& E.L. Schneider. 2000. SEM studies on vessels in fern 18. Montane Cheilanthoid ferns (Pteridaceae) on North America. Aliso 19: 31-39.

Carlquist, S. \& E.L. Schneider. 2001. Vessels in ferns: structural, ecological, and evolutionary significance. Amer. J. Bot. 88: 1-13.

Chaerle, P. \& R.L.L. Viane. 2004. Leaf anatomy and the occurrence of false veins in Asplenium (Aspleniaceae, Pterydophyta). Bot. J. Linn. Soc. 145: 187-194.

D’Ambrogio, A.A. 1986. Manual de técnicas de histología vegetal. Hemisferio Sur, Buenos Aires, Argentina. $83 \mathrm{p}$.

Díaz-Barriga, H. \& M. Palacios-Rios. 1992. Listado preliminar de especies pteridofitas de los estados de Guanajuato, Michoacán y Querétaro, México, p. 1-45. In J. Rzedowski \& G.C. de Rzedowski. Flora del Bajío y de regiones adyacentes. Fascículo Complementario III. Xalapa, Veracruz, México.

Esau, K. 1976. Anatomía vegetal. Omega, Madrid, España. $779 \mathrm{p}$.

Gardner, R.O. 1975. An overview of botanical clearing technique. Stain Technol. 50: 99-105.

Guantay, M.E. \& M. Hernández. 1990. Estudio morfoanatómico de las especies del género Elaphoglossum Schott de Tucumán. Lilloa 38: 73-84.

Hernández H., V. 2003. Anatomía y morfología del complejo Dryopteris patula (Pteridophyta: Dryopteridaceae) en México. Tesis de Maestría, Colegio de Postgraduados, Montecillo, México. 88 p. 
Herrera, M.C.M. 1993. Anatomía de dos especies de Cheilanthes Swartz. Tesis de Maestría, Colegio de Postgraduados, Montecillo, México. 107 p.

Johansen, D.A. 1940. Plant microtechnique. McGraw-Hill, Nueva York, EEUU. 523 p.

Knobloch, I.W. \& D.S. Correll. 1962. Ferns and fern allies of Chihuahua, Mexico. Texas Research Foundation, Texas, EEUU. 198 p.

Kramer, K.U. \& P.S. Green. 1990. Pteridophytes and gymnosperms, p. 101-112. In K. Kubitzki (ed.). The families and genera of vascular plants. Springer, Berlín, Alemania.

Li, R., D. Zhang \& H. Zhang. 1999. Scanning electron microscope observations on the vessels of ferns: Adiantum, Matteuccia and Osmunda from Heilongjiang Province, China. Int. J. Plant Sci. 160: 595-602.

Li, R., X. Yan \& D. Zhang. 2000. Vessel in root and rhizomes of Dryopteris crassirhizoma (Dryopteridaceae) from Heilongjiang Province, China. Amer. Fern J. 90: 24-31.

Lin, B.L. \& C.D. DeVol. 1977. The use of stipe characters in fern taxonomy I. Taiwania 22: 91-99.

Lin, B.L. \& C.D. DeVol. 1978. The use of stipe characters in fern taxonomy II. Taiwania 23: 77-95.

Lucansky, T.W. 1981. Chain ferns of Florida. Amer. Fern J. 71: 101-108.

Mendoza, C.S., L. Zelaya, L. Pacheco \& C. Aguilar. 1998. Anatomía de varias especies del género Diplazium Swartz., p. 4. In VII Congreso Latinoamericano de Botánica. XIV Congreso Mexicano de Botánica, Diversidad y Conservación de los Recursos Vegetales en Latinoamérica. México, DF, México.

Mickel, J.T. 1992. Dryopteris, p. 258-265. In R. McVaugh \& W.R. Anderson (eds.). Flora Novo-Galiciana. Univ. of Michigan, Michigan, EEUU.

Mickel, J.T. \& J.E. Beitel. 1988. Pteridophyte Flora of Oaxaca, Mexico. Mem. N. Y. Bot. Gard. 46: 320-327.

Mickel, J.T. \& A.R. Smith. 2004. The Pteridophytes of Mexico. Mem. N. Y. Bot. Gard. 88: 1-1054.

Moran, R.C. 1987. Monograph of the neotropical fern genus Polybotrya (Dryopteridaceae). III. Nat. Hist. Surv. Bull. 34: 1-138.

Moran, R.C. 1995. Dryopteris, p. 212-214. In R. Moran \& R. Riba (eds.). Flora Mesoamericana, Psilotaceae a Salviniaceae. Instituto de Biología, Missouri Botanical Garden y The Natural History Museum, México, DF, México.
Niklas, K.J. 1992. Plant biomechanics. Chicago, Illinois, EEUU. 607 p.

Ogura, Y. 1972. Comparative anatomy of the vegetative organs of the Pteridophytes. Handbuch der Pflanzenanatomie. Borntraeger, Berlín, Alemania. $502 \mathrm{p}$.

Pacheco, L. \& F. Lorea. 1985. Clave para la identificación de los géneros de Pteridofitas de Veracruz. México. Biotica 10: 157-175.

Pacheco, L. \& R.C. Moran. 1999. Monograph of the neotropical species of Callipteris with anastomosing veins (Woodsiaceae). Brittonia 51: 343-388.

Palacios-Rios, M. 1992. Las Pteridofitas del estado de Veracruz, México. Tesis de Maestría, Universidad Nacional Autónoma de México, México, DF, México. $364 \mathrm{p}$.

Pérez-García, M., B. Pérez-García \& R. Riba. 1995. Anatomía foliar de especies mexicanas de Pleopeltis (Filicales: Polypodiaceae), p. 7. In XIII Congreso Mexicano de Botánica, Diversidad vegetal de México. Cuernavaca, Morelos, México.

Riba, R. 1967. Revisión monográfica del complejo Alsophila artziana Martius (Cyatheaceae). An. Ins. Biol. Univ. Nal. Autón. México. 38. Ser. Bot. 1: 61-100.

Riba, R. \& B. Pérez-García. 1999. Pteridofitas familia Dryopteridaceae, p. 1-48. In R. Riba, N. Esparza, R.Galván, F. Flores \& H. Hernández (eds.). Flora de México. Consejo Nac. Fl. México, México, DF, México.

Rzedowski, J. 1978. Vegetación de México. Limusa, México, DF, México. 432 p.

Saiki, Y., M. Matsumoto \& Y. Mitsuda. 1988. Vascular patterns in the petiole of Aspleniaceae. Proceeding of the ISSP: 273-278.

Schneider, E.L. \& S. Carlquist. 1998a. SEM studies on vessels in ferns 7. Microgramma nitida. An. Ins. Biol. Univ. Nal. Auton. México. Ser. Bot. 69: 1-7.

Schneider, E.L. \& S. Carlquist. 1998b. SEM studies on vessels in ferns 9. Dicranopteris (Gleicheniaceae) and vessel patterns in leptosporangiate ferns. Amer. J. Bot. 85: 1028-1032.

Schneider, E.L. \& S. Carlquist. 1998c. SEM studies on vessels in fern 5. Woodsia scopulina. Amer. Fern J. 88: 17-23.

Schneider, E.L. \& S. Carlquist. 1999a. SEM studies on vessels in ferns 11. Ophioglossum. Bot. J. Linn. Soc. 129: 105-114. 
Schneider, E.L. \& S. Carlquist. 1999b. SEM studies on vessel in ferns 13. Nephrolepis. Amer. J. Bot. 89: 171-177.

Schneider, E.L. \& S. Carlquist. 2000. SEM studies on vessels in ferns 19. Marsilea. Amer. Fern J. 90: 32-41.

Sen, U. \& E. Hennipman. 1981. Structure and ontogeny of stomata in Polypodiaceae. Blumea 27: 175-201.

Sen, U. \& D. Mittra. 1966. The anatomy of Cystodium. Amer. Fern J. 56: 97-101.

Silva, P.D., L. Pacheco M. \& S. Mendoza. 2001. Morfología y anatomía foliar de dos géneros Diplazium y Callipteris (Woodsiaceae), CD-ROM. In XV Congreso Mexicano de Botánica. Querétaro, México.

Smith, A.R. 1981. Flora of Chiapas, p. 101-103. In D.S. Breedlove (ed.). California Academy of Sciences, California, EEUU.

Tryon, R.M. \& A.F. Tryon. 1982. Ferns and allied plants with special reference to tropical America. Springer, Nueva York, EEUU. 857 p.
Velasco, R.C. 1971. Estudio anatómico comparativo de dos especies del género Marattia (Marattiales, Pteridophyta). Tesis profesional, Universidad Nacional Autónoma de México, México, DF, México. 63 p.

Velásquez, F.E.M. 1983. Anatomía comparada de dos especies del género Tectaria Cav. (Filicales, Pteridophyta). Tesis de Licenciatura, Universidad Nacional Autónoma de México, México, DF, México. 62 p.

White, R.A. 1974. Comparative anatomical studies of the ferns. Ann. Mo. Bot. Gard. 61: 379-387.

Zavaro, P.C.A., C. Sánchez \& J. Bozán. 1995. Anatomía de las especies cubanas del género Thelypteris Schmidel, subgénero cyclosorus Morton. Fontqueria 42: $195-198$

Zhang, X.C. \& H.P. Nooteboom. 1998. A taxonomic revision of Plagiogyriaceae (Pteridophyta). Blumea 43: 401-469.

Zlotnik, E.A. 1991. Anatomía de Polypodiaceae s. str. Tesis de Maestría, Universidad Nacional Autónoma de México, México, DF, México. 103 p. 
